\title{
Ankle Fractures: A Literature Review of Current Treatment Methods
}

\author{
Rohit Singh1, Tamer Kamal1,2, Nick Roulohamin', Gopikanthan Maoharan1, Bessam Ahmed1, \\ Peter Theobald ${ }^{3}$ \\ ${ }^{1}$ Robert Jones and Agnes Hunt, Oswestry, UK \\ ${ }^{2}$ Orthopaedic Department, Cairo University, Giza, Egypt \\ ${ }^{3}$ Cardiff school of Engineering, Cardiff University, Cardiff, UK \\ Email: Rohitsing@hotmail.com
}

Received 16 September 2014; revised 31 October 2014; accepted 16 November 2014

Copyright (C) 2014 by authors and Scientific Research Publishing Inc.

This work is licensed under the Creative Commons Attribution International License (CC BY).

http://creativecommons.org/licenses/by/4.0/

(c) (i) Open Access

\begin{abstract}
Ankle fracture is one of the most common lower limb fractures for they account for $9 \%$ of all fractures representing a significant portion of the trauma workload. Ankle fractures usually affect young men and older women, however, below the age of 50; ankle fractures are the commonest in men. Two commonly used classification systems for ankle fractures include the danis weber AO classification and the Lauge-Hansen classification. There is biomechanical evidence that posterior non-locking plates are superior in stability than laterally placed plates; however there is little clinical evidence. There are several different methods of ankle fracture fixation, however the goal of treatment remains a stable anatomic reduction of talus in the ankle mortise and correction of the fibula length as a $1 \mathrm{~mm}$ lateral shift of the talus in the ankle mortise reduces the contact area by $42 \%$, and displacement (or shortening) of the fibula more than $2 \mathrm{~mm}$ will lead to significant increases in joint contact pressures. Further research both biomechanically and clinically needs to be undertaken in order to clarify a preferable choice of fixation.
\end{abstract}

\section{Keywords}

Ankle Fracture, Review, Current Treatment, Classification, Management

\section{Introduction}

Ankle fractures are one of the most common lower limb fractures [1]; they account for 9\% of all fractures [2], representing a significant portion of the trauma workload [3]. The annual incidence of ankle fracture is between 107 and 184 per 100,000 persons [4]-[6], and around $2 \%$ of ankle fractures are open fractures [4]. Ankle frac- 
tures usually affect young men and older women [7], however, below the age of 50; ankle fractures are the commonest in men. After this age, females become predominant. Alcohol and slippery surfaces are each involved in nearly a third of the cases [6]. The most common causes of ankle fractures are twisting injuries and falls, followed by sports injuries [4] [5]. Diabetes mellitus and obesity are associated with fractures in middleaged and older adults [5]. Most fractures are associated with ligament injuries, and the magnitude and direction of the deforming force applied to the ankle joints directly correlate to the fracture pattern [8].

\section{Joint Anatomy and Biomechanics}

The ankle joint is a hinge joint, the lower end of the tibia and its medial malleolus, together with the lateral malleolus of the fibula and the inferior transverse tibiofibular ligament form a deep recess (mortise) to articulate with the body of the talus, the ankle joint has a strong medial collateral (deltoid) ligament which is triangular in shape, it is made up of the tibionavicular, tibiocalcaneal and tibiotalar (anterior and posterior) ligaments (Figure 1), injury to the deltoid ligament is commonly associated with distal fibula fractures [8].

The lateral ligament has 3 parts, the anterior talofibular ligament the posterior talofibular ligament and the calcaneofibular ligament, the lateral ligament is commonly injured with inversion sprains (Figure 2) [8].

The inferior tibio fibular ligament is usually considered a syndesmosis, consisting of the anterior and posterior tibio fibular ligaments and the interosseous ligament.

The ankle joint receives its blood supplied from the anterior and posterior tibial and fibular arteries. The joint is innervated by branches from deep fibular, saphenous, sural and tibial nerves [8].

Factors maintaining stability: passive stability is mainly achieved by the medial and lateral ligament complexes, tibiofibular ligaments, tendons crossing the joint, bony contours and capsular attachments. Dynamic stability is usually conferred by gravity, muscle action and ground reaction forces. Stability requires the continuous action of soleus assisted by gastrocnemius, it increases on leaning forward and decreases on leaning backwards [8]. The posterior malleolus acts as a restraint against posterior translation of the talus, and fractures involving approximately $25 \%$ of the articular surface will result inposterior instability [9].

The ankle joint is subjected to enormous forces across a relatively small surface area of contact, with up to 1.5 times body weight with gait and greater than 5.5 times bodyweight with more strenuous activity. Maintaining congruency of the ankle joint is therefore critical to the long-term viability of the ankle [9].

\section{Classification}

The first classification system for ankle fractures, developed by Percival Pott, describes fractures in terms of the number of malleoli involved, thus dividing injuries into unimalleolar (70\%), bimalleolar (23\%) and trimalleolar (7\%) [4], although easy to use, with good intra-observer reliability, it does not distinguish between stable and unstable injuries [3].

There are two other commonly used classifications systems:

(1) Danis-Weber AO Classification

It was introduced by Danis in 1949, modified by Weber in 1966 then adopted by the AO group.

Three types are recognized based largely upon the level of the fibula fracture and the associated level of syndesmosis disruption (Figure 3):

A) Characterized by a transverse fibula fracture at or below the ankle joint and no syndesmotic injury;

B) Involves a spiral fibula fracture beginning at the level of the joint and a partial syndesmotic injury;

C) The fracture is proximal to the ankle joint with associated disruption of the syndesmosis to this level [10].

This classification is attractive for clinical because it is simple. The disadvantage of this system is that it does not incorporate a staging system to allow for the grading of injuries falling under one heading in terms of severity [11].

(2) Lauge-Hansen Classification

In 1950 Lauge-Hansen devised a classification of ankle injuries, as a result of both cadaveric dissections of experimentally produced fractures, and clinical and radiological examinations [9]. The basis of this classification is on the position of the foot and the deforming force at the time of injury. This was found experimentally by fixing fresh cadaver feet to boards and subjecting them to different stresses.

Lauge-Hansen established four basic types of ankle injuries:

a) pronation-abduction; 


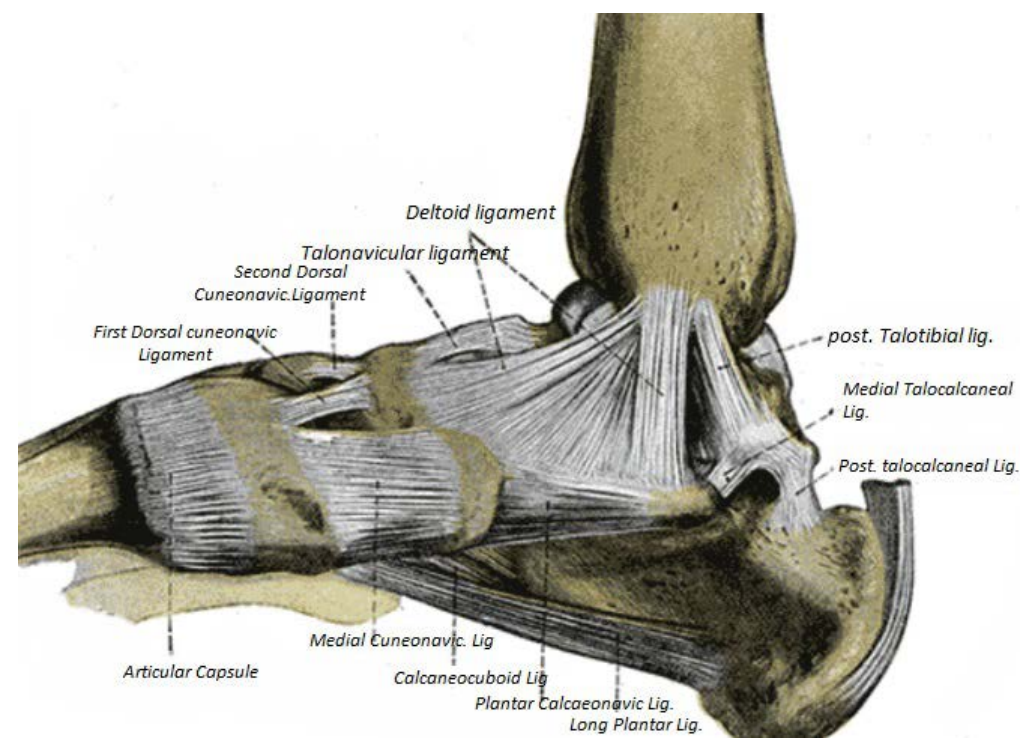

Figure 1. Ligaments of the medial aspect of the foot [8].

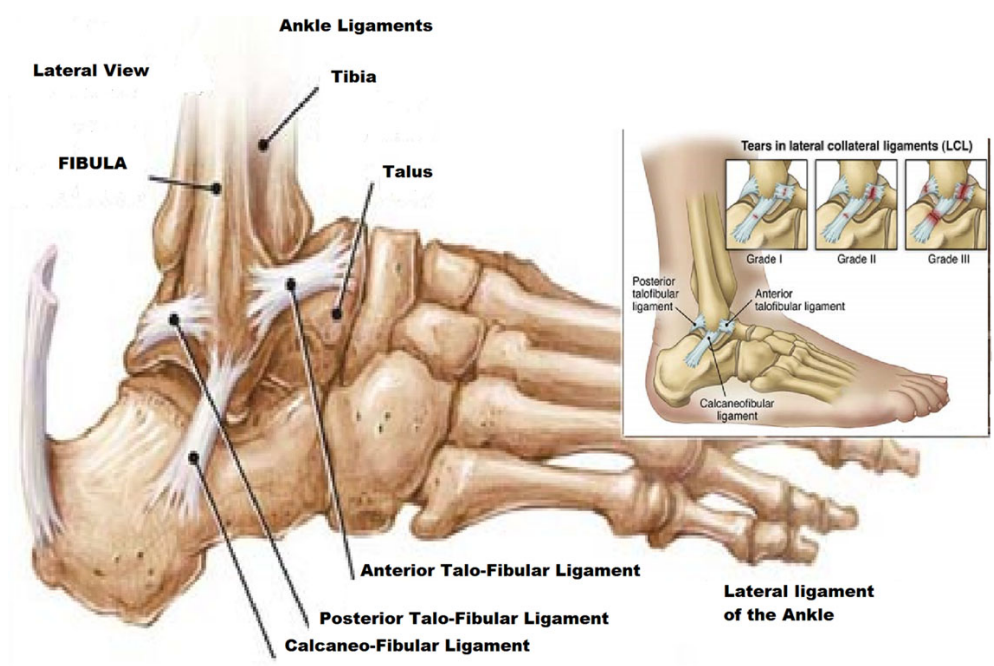

Figure 2. The lateral ligament complex [8].

b) pronation-lateral rotation (eversion);

c) supination-adduction;

d) supination lateralrotation (inversion).

The first term of this dual designation refers to the position of the foot at the time of injury; the second refers to the deforming force.

He discovered that with each type of force to the ankle, injury to the ligaments and bones usually occurs in a predictable sequence (Figure 3). His experimental work has been widely accepted and serves as a basis for evaluating ankle injuries [10] [11]

(1) Supination/Abduction: resulting in a transverse fracture of the distal fibula, then an oblique fracture of the medial malleolus [8].

(2) Supination/external rotation: The most common pattern of ankle fractures [12], the first structure to rupture is the anterior tibiofibular ligament followed by a spiral fibula fracture, posterior malleolus fracture then medial malleolus fracture/or deltoid ligament rupture.

(3) Pronation/abduction: there will be a transverse medial malleolus fracture, followed by rupture of the anterior and posterior tibiofibular ligaments and the interosseous ligament and then an oblique fibular fracture.

(4) Pronation/external rotation: Leads to medial malleolus fracture (or deltoid ligament rupture) followed by 


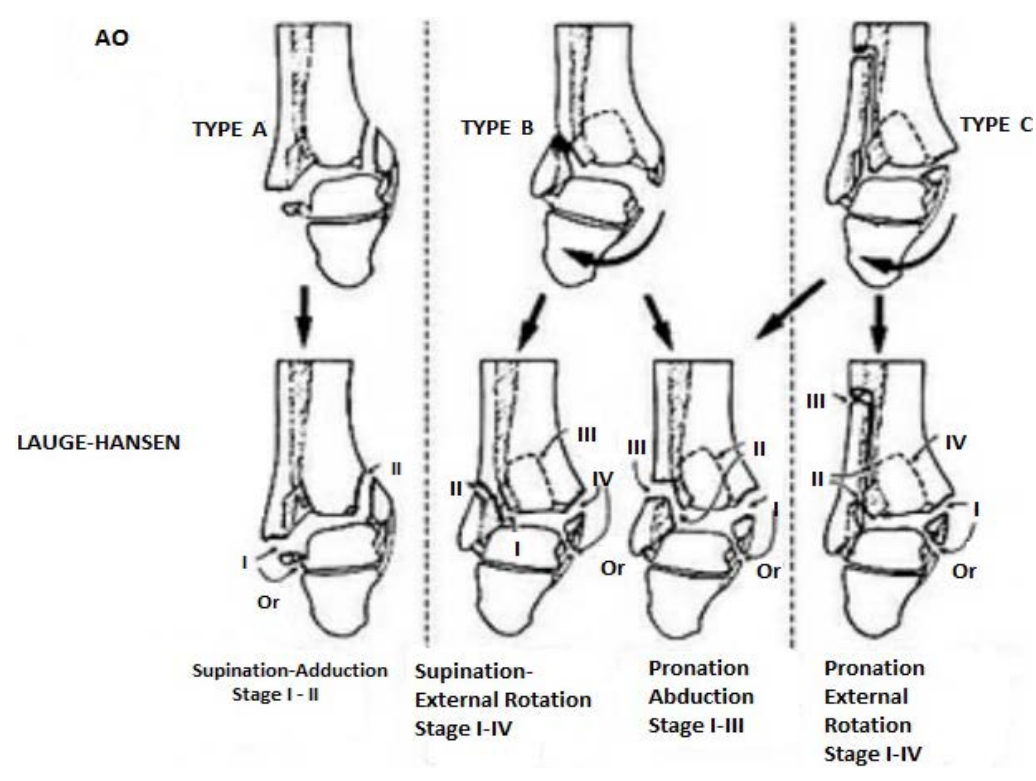

Figure 3. Classification of Ankle fractures [8].

rupture of the anterior inferior tibiofibular ligament, interosseous ligament, interosseous membrane, high fibula fracture, then a posterior malleolus fracture [8].

\section{Diagnosis}

Ankle fractures usually result from torsional forces and present typically with tenderness, swelling, deformity and inability to weight-bear. However, beware the non-displaced ankle fracture presenting post-injury with minimal swelling and no deformity. The history of the injury normally gives a good indication of the mechanism of fracture and can assist in decision making regarding management. The physical examination of the ankle must also involve a thorough assessment of the foot.

The assessment looks at any open wounds, the condition of the skin, neurovascular status before and after any reductions and palpation for any bony or soft tissue tenderness. Physical examination revealing medial tenderness has been shown to have poor positive predictive value for significant deltoid injury [12]. It is vital to assess for proximal fibular tenderness in order to rule out Maisonneuve fractures [13]. Soft tissue palpation of all the ligamentous structures should be performed. These include the anterior talofibular ligament, the posterior talofibular ligament, the calcaneofibular ligament, the deltoid ligament complex and the anterior tibiofibularsyndesmosis. The range of motion should be assessed actively and passively: dorsiflexion, plantarflexion, inversion and eversion. These should be compared bilaterally. Normal passive dorsiflexion is 10-15 degrees, plantarflexion 50-70 degrees, inversion 40 degrees and eversion is 10 degrees. Finally, there are some special assessment tests that can be performed but their sensitivity is questionable [14].

The external rotation stress test (Kleiger test) involves stabilising the distal tibia whilst externally rotating the foot. This external rotation leads to the talus applying pressure to the lateral malleolus, causing widening of the tibiofibular joint. Increased widening or pain when compared to the normal ankle indicates a syndesmosis injury or a deep deltoid ligament injury. Compression of the tibia and fibula proximal to the ankle joint, the squeeze test, will also elicit pain at the syndesmosis if it is injured [14].

Anterior tibiofibular ligament injury can be evaluated by the anterior drawer test for the ankle. The distal tibia is stabilised, the ankle is maintained in 10 degrees of plantarflexion and the heel is drawn anteriorly. The same manoeuvre is performed on the normal ankle and pain and instability indicate ligament injury. A forward shift of more than $8 \mathrm{~mm}$ on a lateral radiograph is considered diagnostic for an anterior tibiofibular ligament tear [15].

The talar tilt test examines the integrity of the lateral ankle ligaments, mainly the calcaneofibular ligament. It is performed by stabilising the distal tibia and applying an inversion force to a slightly dorsiflexed foot. The test is positive if comparison of the medial and lateral aspects demonstrates a difference of greater than $25 \%$. Van Dijk et al. found better specificity (84\%) and sensitivity (96\%) of the drawer test and talar tilt test if examination 
was delayed for 4 - 7 days, compared with examination within 48 hours (specificity 33\%, sensitivity 71\%) [15] [16].

\section{Radiological Evaluation and the Role of the Stress View}

There are a few indications for obtaining radiographs of the ankle. The best known tool to indicate the requirement for X-rays is Ottawa ankle rules [17]. There are other indications like a patient who cannot communicate (altered mental state, alcohol intoxication); persistent pain and swelling for 7 - 10 days post-injury and anytime the history and examination fail to provide sufficient information. These rules generally apply to patients with skeletal maturity (55 years or older).

The Ottawa ankle rule project demonstrated that more than $95 \%$ of patients with ankle injuries had radiographic examinations, but that $85 \%$ of the films showed no fractures. A group of Ottawa emergency physicians developed two rules to identify clinically important fractures of the malleoli and the midfoot. Use of these rules reduced radiographic examinations by $28 \%$ for the ankle and $14 \%$ for the foot [17].

A systematic review by Bachmann et al supports the Ottawa ankle rules as an accurate instrument for excluding fractures of the ankle and mid-foot. The instrument has a sensitivity of almost $100 \%$ and a modest specificity, and its use should reduce the number of unnecessary radiographs by $30 \%-40 \%$ [18]. The Ottawa ankle rules also appear to be a reliable tool to exclude fractures in children older than 5 years presenting with ankle and mid-foot injuries, with a significant decrease in X-ray use and a low likelihood of missing a fracture [19] [20].

The Ottawa ankle rules state that X-rays are only required if there is any pain in the malleolar zone and any one of the following:

- Bone tenderness along the distal $6 \mathrm{~cm}$ of the posterior edge of the tibia or tip of the medial malleolus ;

- Bone tenderness along the distal $6 \mathrm{~cm}$ of the posterior edge of the fibula or tip of the lateral malleolus;

- An inability to bear weight both immediately and in the emergency department for four steps.

Ankle fracture radiographs account for a significant percentage of plain radiographs in the Accident and Emergency department. In instances of suspected ankle bony injury, three views of the ankle joint should be obtained to evaluate for fractures and talar subluxation or dislocation. Brandser et al. emphasized the necessity of obtaining radiographs in anteroposterior (AP) view, mortise view, and lateral view [21].

The AP view is taken with the patient supine and foot in neutral position. The sole of the foot is perpendicular to the cassette and the heel rests on the cassette. The X-ray beam is directed centrally towards the midpoint of the malleoli. The entire fibula is included if there is lateral tenderness superior to the joint line. AP view is used to evaluate the medial and lateral malleoli, anterolateral tibia, the proximal fibula and tibiofibular clear space. It demonstrates articular congruity, malleolar length, syndesmotic integrity and talar shift. Tibiofibular overlap on an AP view indicates proper syndesmotic relationship. The space between the medial fibular wall and the tibialincisural surface, the tibiofibular clear space, should be less than $5 \mathrm{~mm}$. The anterior tibial tubercle should overlap the fibula by at least $6 \mathrm{~mm}$ or $42 \%$ of the fibular width. The Shenton line, drawn from the Wagstaffe tubercle of the fibula to the medial malleolus should pass through the tibial plafond. Incongruency of this line indicates fibular shortening [22] [23].

The lateral ankle view is obtained with the lateral border of the foot against the cassette and the ankle in slight dorsiflexion. Sometimes a horizontal beam needs to be used. The lateral ankle view demonstrates fibular fracture patterns, posterior tibial tuberosity fractures and anterior or posterior displacement of the talus. The dome of the talus should be centred under, and be congruous with the tibial plafond [22] [23].

For the mortise view, the entire leg and foot are internally rotated to 15 - 20 degrees, the ankle is dorsiflexed and the X-ray beam is centred on the intermalleolar line and perpendicular to this. This view is used to evaluate for talar shift, fibular length and rotation, talocrural angle, tibiofibular clear space and medial clear space. The medial clear space is the distance between the medial malleolar articular surface and the medial edge of the talus. It is normally $4 \mathrm{~mm}$ and widening indicates fracture and or injury to the syndesmosis. The talocrural angle is the angle subtended by a line drawn parallel to the tibial plafond articular surface and a line connecting the tips of the malleoli and is normally 4 - 11 degrees. Alternately it can be measured as the angle between a line perpendicular to the tibial plafond articular surface and a line joining the tips of the malleoli. This is measured at 83 $+/-4$ degrees. The talocrural angle must be within 2 - 5 degrees of that on the unaffected side and a greater difference indicates fibular shortening. The dime test, an unbroken curve connecting the recess in the distal tip of the fibula and the lateral process of the talus, helps evaluate fibular shortening on the mortise view [22] [23].

Additional special views can be requested to evaluate areas of concern. These views include oblique views, 
stress views and weight-bearing views. Oblique views provide additional information about the ankle mortise, talar dome and the malleoli. The internal oblique view provides additional information about the lateral malleolus and the external oblique view about the medial malleolus.

In patients with suspected soft tissue injuries, stress views provide information on ligamentous stability. This involves stressing the ankle into supination and external rotation and then exposing it to X-rays. The external rotation stress view evaluates syndesmotic and deep deltoid ligament instability by measuring the difference in width of superior clear space between the medial and lateral sides of the joint. This difference should be less than $2 \mathrm{~mm}[21]$ [24].

CT scanning and MRIs are not needed for the evaluation of most ankle fractures. CT scans may be used to better define pilon fractures and triplanar fractures. MRIs can demonstrate additional injuries in children with Salter-Harris fractures, and may also be used to evaluate occult injuries of the talar dome and soft tissue injuries [25]. Syndesmotic injury as predicted by the Lauge-Hansen fracture classification correlate well with MRI findings. With MRI the extent of syndesmotic injury and therefore fracture stage can be assessed more accurately compared to radiographs [25] [26]. Hsu et al found ultrasound imaging to be useful for identifying ligament injuries in patients with inversion ankle sprains [27]. The one disadvantage of ultrasounds is that it is operator dependent. Bone scintigraphy can be used to look for occult injuries, especially injuries of the talar dome [23].

\section{Intra-0perative Imaging}

Frankhauser and Musgrave performed and published a retrospective study in 1998 to determine whether three or two radiographic views are necessary for intra-operative evaluation of low energy, rotational ankle fracture reduction and fixation. They concluded that fracture reduction and fixation can be assessed adequately with lateral and mortise views. The AP view can be eliminated from the standard radiographic protocol, potentially resulting in cost savings [28].

Intra-operative stress testing is required for the detection of syndesmosis instability following an ankle fracture. A cadaveric study published in2009 compared two stress tests for the detection of syndesmotic injury at the site of ankle fractures on stress radiographs. The lateral stress test appeared to be superior to the external rotation stress test in this cadaver model [29].

Another study published in 2012 states that following open reduction and internal fixation of an ankle fracture, the correct position of the syndesmosis cannot be evaluated reliably with the use of conventional radiographs or intra-operative fluoroscopy. They believed that any syndesmotic injury treatment should include intra-operative three-dimensional imaging or at least a post-operative CT scan [30].

Another study in 2003 suggested that arthroscopy of the ankle is indispensable for the accurate diagnosis of a tear of the tibiofibular syndesmosis. In comparison with arthroscopy, the sensitivity, specificity and accuracy were $44.1 \%, 100 \%$ and $63.5 \%$ for standard AP radiography and $58.3 \%, 100 \%$ and $71.2 \%$ for mortise radiography [31].

Lui et al. performed a comparative study in 2005 that indicates that ankle arthroscopy excels intra-operative stress radiography in detecting syndesmosis disruption. It also provides assessment of different planes of instability and assists anatomic reduction of the syndesmosis. Intra-operative radiography still does play an important role in assessing fracture reduction and proper restoration of fibular length and longitudinal orientation of the syndesmosis [32].

\section{Treatment}

Ankle fractures can be treated conservatively or surgically depending on the type of fracture and the surgeon's opinion, the question of whether surgery or conservative treatment should be used for ankle fractures remains controversial [33].

Surgical treatment involves the reduction (if displaced) of the fractured parts and fixation using various devices such as metal plates, screws, tension bands or external fixation. These operative techniques aim to provide anatomical restoration and immediate stability, which facilitates earlier mobilisation. However, all surgery carries the risk of complications such as wound infection, pulmonary embolism, implant or fixation failure, mortality, amputation and reoperation [34].

With early mobilisation and postoperative exercises these adverse effects may be prevented. If surgical treatment can protect or accelerate the bone healing process by securely stabilising the fracture, it can also reduce 
recovery and 'back-to-work-time' and therefore indirect costs to society. This may not be the case for older people with osteoporosis because the porosity of their bones may increase the risk of fixation failure and thus preclude early mobilisation [34]. There is limited evidence supporting early commencement of weight-bearing and the use of a removable type of immobilisation to allow exercise during the immobilisation period after surgical fixation [34].

Conservative treatment involves manipulating the fractured bone to reposition the ankle mortise, followed by immobilisation of the ankle in a plaster cast for several weeks [7]. For conservative treatment, immobilisation of the fractured parts is generally considered to be important for bone healing. However, immobilisation can lead to muscle atrophy, cartilage degeneration, and a stiff, painful and swollen joint. Also, conservative treatment can lead to secondary displacement, which generally requires surgery, painful non-union and prolonged immobilisation [7] [33].

However, Wei et al. followed up 19 patients over 20 years and showed that that bi- and trimalleolar fractures can be treated non-operatively if an anatomic reduction is maintained and closely followed [35].

\section{Rationale behind ORIF of Ankle Fractures}

The key to open reduction and internal fixation for displaced ankle fractures is to restore tibiotalar congruency, as Ramsey and Hamilton in 1976 showed that a $1 \mathrm{~mm}$ lateral shift of the talus in the ankle mortice reduces the contact area by $42 \%$ [36]. Further studies have shown that posterior malleolus fractures involving more than 33\% leads to a significant loss of tibiotalar contact [37]. However, DeSouza, 1985 showed $90 \%$ satisfactory results could be obtained even if up to $2 \mathrm{~mm}$ of lateral displacement was present [38].

Generally open reduction and internal fixation is recommended in young patients if there is over $1 \mathrm{~mm}$ displacement or 2 degrees talartilt [39]. With elderly patients; $2 \mathrm{~mm}$ of displacement is deemed acceptable [39]. One must always take into account the ambulatory needs of the patient and judge treatment accordingly.

There is a reduction in the incidence of arthrosis in patients where an anatomical reduction has been achieved as prospective trials shows higher total ankle scores in those that are treated operatively [40]. However, this incidence increases with severity of injury [40]. Further studies demonstrated that degenerative change in the ankle of $10 \%$ of anatomically fixed fractures compared with $85 \%$ if not adequately reduced, and these changes apparent within 18 months [39] [41].

It is estimated that $10 \%$ of all ankle fractures are associated with syndesmotic disruption [42], syndesmotic screw fixation is recommended when there is a tibiofibular diastasis, a Maisonneuve fracture, or syndesmotic instability after fixation of distal tibia-fibula fractures. There is currently no consensus about the optimum method of stabilization, position of the ankle during implant placement, weight-bearing restrictions, or need for and timing of implant removal. Screw fixation currently remains the gold standard and should be placed as position screws rather than as lag screws. Reduction of the syndesmosis must therefore be obtained before implant placement - a large pelvic clamp is invaluable in obtaining the reduction [42].

Whether or not the syndesmosis screw should be removed prior to weight-bearing is still debated, there is paucity in randomized controlled trials on the absolute need for removal of the syndesmotic screw. However, current literature suggests that it might be reserved for intact screws that cause hardware irritation or reduced range of motion after 4 - 6 months [43].

Posterior malleolus fractures need to be fixed if there is over $25 \%$ of the articular surface is involved. This is often underestimated on lateral radiographs. Restoration of fibular length should indirectly reduce the posterior malleolus, the fragment can be provisionally stabilized with 1.6- $\mathrm{mm}$ Kirschner wires and definitive cortical lag screw fixation placed percutaneously. This could be done either by anterior to posterior screw fixation, starting anteromedially and aiming posterolaterally, or posterior to anterior fixation can be used, however, limb positioning typically makes this option technically demanding [9].

Isolated fractures of the medial malleolus can obtain high rates of union and good functional results with conservative treatment. Operation should be reserved for bi- or trimalleolar fractures, open fractures, injuries which compromise the skin or those involving the plafond or for patients who develop painful non-union [40].

\section{Post-Operative Management}

In studies comparing the effect of early movement compared to immobilisation and weight bearing versus nonweight bearing, the conclusion is that there is no difference in the final result whichever regime is used [44]- 
[49].

\section{Prognosis}

In general, the results following an anatomic reduction of displaced ankle fractures are good. Post traumatic arthritis has been described in $10 \%$ of patients despite an anatomic reduction [41], most likely as a result of chondral injury sustained at the time of initial injury. One arthroscopic study found $79 \%$ of patients to have some degree of chondral injuries, especially in patients with Weber $\mathrm{C}$ fractures/Pronation external rotation injuries [50]. Although some degree of stiffness is to be anticipated, most patients resume full activities following healing of these fractures [3] [47] [48].

\section{Comparison of Different Fixation Techniques}

There are several different methods of ankle fracture fixation, however the goal of treatment remains a stable anatomic reduction of talus in the ankle mortise and correction of the fibula length as a $1 \mathrm{~mm}$ lateral shift of the talus in the ankle mortise reduces the contact area by $42 \%$ [37], and displacement (or shortening) of the fibula more than $2 \mathrm{~mm}$ will lead to significant increases in joint contact pressures [51].

Fibular fixation can be achieved with a number of different techniques, including intramedullary fixation, interfragmentary screw fixation, and plate fixation [52].

Fixation with one or two lag screws can only be used in younger patients with long oblique fractures. The fibular fracture has to be sufficiently oblique, non-comminuted, and non-osteoporotic, then sufficient fixation can be achieved with only two lag screws. Also the oblique fracture should be longer than two times the diameter of bone in order for the fracture to be fixed with 2 lag screws alone. When these criteria are not met, then application of a lateral or posterior plate will be necessary [53].

The lag screw technique involves placement of one or more screws across a fracture or osteotomy site to achieve interfragmentary compression. The direction along which the compression acts (inclination of lag screw) must coincide with the perpendicular axis of the fracture surface. It has been shown that sliding of the fracture will occur if the compression is applied at an angle greater than $20 \mathrm{deg}$ in relation to the axis perpendicular to the fracture surface [53].

The advantages of lag screw alone are that it allows for a smaller incision, the hardware is not prominent and usually does not have to be removed, and the lag screws will not interfere with syndesmotic screws (if they are needed) [53].

When the criteria for lag screw fixation are not met, then lateral or posterior plate application with and without lag screw insertion can be used. The disadvantages of lateral plating are that prominent lateral screws may cause symptoms or wound necrosis, possibility of distal intra-articular screw insertion, and on the contrary, there is the possibility of inadequate fixation if distal screws are too short. Also it may not allow adequate fixation in osteoporotic bone, and may interfere with syndesmotic screw insertion (especially when two syndesmoic screws are to be used) [54].

The position of the plate depends on the level of fracture, condition of overlying soft tissues, and the extent of comminution. A posteriorly positioned plate allows use of a thicker, stronger plate, less risk of wound slough, and the distal screws can obtain better purchase since they engage a thicker part of distal fibula and engage 2 cortices without risk of joint penetration [54]. However, the plate can irritate peroneal tendons and will not allow syndesmosis screw insertion [55] [56].

When performing a literature review of the different techniques of ankle fracture fixation, there was conflicting evidence:

Schaffer and Manoli performed a biomechanical analysis of lateral plates compared with posteriorly placed plates for stabilized short oblique fibula fractures. They performed stiffness measurements and cyclic load to failure and found that the lateral plate was inferior, failing at $64 \%$ of initial torque, whereas the posterior plate failed at $77 \%$ [54].

Minihane et al. evaluated 18 fibula fractures created by an oblique saw cut and ligamentous sectioning. Their fractures were fixed with either a lateral locking plate with an independent lag screw or a posterolateral antiglide plate with a lag screw through the plate. Torsonial load to failure was performed. Interestingly, the posterior non-locking plate was still biomechanically superior to the lateral locking plate in torque to failure and stiffness. They believed that the posterior anti-glide plate shared the deforming forces transferred from the distal 
fragment, requiring the plate to deform prior to failure. In contrast, the strength of the lateral locking plate construct was dependent on the screw-bone interface and failed with screw cut-out through soft bone. Furthermore, the posterior plate benefited from posteriorly to anteriorly directed bicortical screw fixation, whereas only unicortical fixation with shorter working lengths was possible through the lateral plate secondary distal tibiofibular articulation [55].

Although posterior plating appears to be superior biomechanically, there are some clinical limitations to the technique. Weber and Krause treated 70 patients with posterior plating and reported that 30 patients $(43 \%)$ required hardware removal. When evaluated intraoperatively, 9 of these 30 patients had peroneal tendon lesions. They correlated these findings with prominent screw heads and placement of the most distal screw [56].

When comparing posterior and lateral plating techniques clinically, Lamontagne et al. found no statistically significant difference in operating room time, tourniquet time, and hospital stay. There were also no significant differences found in functional score, infection rate, screw loosening, or loss of reduction. There was a slightly higher incidence of hardware irritation, hardware removal, and wound dehiscence associated with lateral plating, although the difference was not statistically significant. Clearly, both plating techniques appear to be limited by local symptoms related to irritation of nearby soft tissue structures [57].

Koval et al. performed a retrospective clinical study and biomechanical laboratory study supporting the use of plate fixation augmented with intramedullary Kirschner wires for the treatment of comminuted and osteopenic fibula fractures in the elderly [58].

Panchbhavi et al. performed a biomechanical labaroty study using fresh-frozen cadaver legs where identical supination-external rotation type II ankle fracture was created. This study advocated internal fixation of an osteoporotic lateral malleolar fracture using a locking plate and screws provided a construct comparable in strength to that augmented with calcium sulfate-calcium phosphate graft and/or tibia-pro-fibula screws [59].

Several studies each compared a different pair of surgical methods including lateral plating versus antiglide plating which were class of evidence class of evidence (CoE) III, rigid internal fixation versus internal splintage with K-wires (CoE III), power-driven staples versus a routine method (CoE II)(88) and rush rods versus AO plates (CoE I) [60]-[62].

Power-driven staples versus routine methods: a significant decrease in average operating time was observed with no significant differences in union or complication rates [60]-[62].

Rush rods with AO plates: no statistically significant differences were seen, but rush rod patients did have slightly higher union rates and rates of good or fair functional results as well as a much shorter time to full weight bearing (six versus twelve weeks) [61].

Lateral versus anti-glide plating: no statistically significant differences in rates of specific complications and no general pattern were observed between groups [57].

Rigid internal fixation versus internal splinting with K-wires: none of the results were statistically significant. It may be of potential clinical importance to note that those treated by rigid fixation were more likely to be walking, pain free (55\% versus $31 \%$ ), more likely to have good joint movement ( $62 \%$ versus $27 \%$ ), less likely to have ankylosed joints or arthrodesis ( $0 \%$ versus $15 \%)$, less likely to have a deep infection (11\% versus $23 \%)$ [62].

\section{Conclusions}

Ankle fractures are one of the most common lower limb fractures [1], representing a significant portion of the trauma workload [3]. Most fractures are associated with ligament injuries, the direction and nature of the force applied to the ankle joints correlating to the pattern of the fracture and ligament injury [8]. Non-operative treatment with immobilisation can provide satisfactory results if anatomical reduction is maintained and closely followed [35], however cast immobilisation can also lead to muscle atrophy, cartilage degeneration, and a stiff, painful and swollen joint. Surgical treatment involves open reduction (if displaced) and internal fixation of the fractured parts using various fixation devices such as metal plates, screws, tension bands or external fixation. These operative techniques aim to provide anatomical restoration and immediate stability, which facilitates earlier mobilisation. All surgery carries the risk of complications such as wound infection, pulmonary embolism, implant or fixation failure, mortality, amputation and reoperation. Management after surgery may also include the use of a plaster cast [34].

When performing a literature review of different techniques of ankle fracture fixation, there is conflicting evidence with two biomechanical studies showing that posterior non-locking plates are superior in stability than laterally placed plates [54] [55], however there is little clinical evidence [57]. There is also conflicting evidence 
regarding complication and failure rates comparing the two plating techniques, with increased peroneal tendon lesions with posterior placed plates [56] [57]. Lag screw fixation is a very popular method of ankle fracture fixation for Weber B spiral oblique fractures, however the fibular fracture has to be sufficiently oblique, non comminuted and non-osteoporotic. There are no biomechanical studies comparing lag screw with neutralisation plates compared with locking plates for Weber B supination external rotation ankle fractures.

In general, the results of ankle fracture fixation are good compared with non-operative treatment with regard to post traumatic arthritis [10].

\section{References}

[1] Lash, N., Horne, G., Fielden, J. and Devane, P. (2002) Ankle Fractures: Functional and Lifestyle Outcomes at 2 Years. ANZ Journal of Surgery, 72, 724-730. http://dx.doi.org/10.1046/j.1445-2197.2002.02530.x

[2] Court-Brown, C.M. and Caesar, B. (2006) Epidemiology of Adult Fractures: A Review. Injury, 37, 691-697. http://dx.doi.org/10.1016/j.injury.2006.04.130

[3] Bugler, K.E., White, T.O. and Thordarson, D.B. (2012) Focus on Ankle Fractures. The Journal of Bone and Joint Surgery, 94, 1107-1112.

[4] Court-Brown, C.M., McBirnie, J. and Wilson, G. (1998) Adult Ankle Fractures-An Increasing Problem? Acta Orthopaedica Scandinavica, 69, 43-47. http://dx.doi.org/10.3109/17453679809002355

[5] Daly, P.J., Fitzgerald Jr., R.H., Melton, L.J, and Ilstrup, D.M. (1987) Epidemiology of Ankle Fractures in Rochester, Minnesota. Acta Orthopaedica Scandinavica, 58, 539-544. http://dx.doi.org/10.3109/17453678709146395

[6] Jensen, S.L., Andresen, B.K., Mencke, S. and Nielsen, P.T. (1998) Epidemiology of Ankle Fractures: A Prospective Population-Based Study of 212 Cases in Aalborg, Denmark. Acta Orthopaedica Scandinavica, 69, 48-50. http://dx.doi.org/10.3109/17453679809002356

[7] Donken, C.C.M.A., Al-Khateeb, H., Verhofstad, M.H.J. and van Laarhoven, C.J.H.M. (2012) Surgical versus Conservative Interventions for Treating Ankle Fractures in Adults. The Cochrane Database of Systematic Reviews, Published Online. http://dx.doi.org/10.1002/14651858.CD008470

[8] Gray's Anatomy. 40th Edition.

[9] Michael, P. and Clare, M.D. (2008) A Rational Approach to Ankle Fractures. Foot and Ankle Clinics, 13, 593-610. http://dx.doi.org/10.1016/j.fcl.2008.09.003

[10] Arimoto, H.K. and Forrester, D.M. (1980) Classification of Ankle Fractures: An Algorithm. American Journal of Roentgenology, 135, 1057-1063. http://dx.doi.org/10.2214/ajr.135.5.1057

[11] Harper, M.C. (1992) Ankle Fracture Classification Systems: A Case for Integration of the Lauge-Hansen and AODanis-Weber schemes. Foot Ankle, 13, 404-407.

[12] Gougoulias, N., Khanna, A., Sakellariou, A. and Maffulli, N. (2010) Supination-External Rotation Ankle Fractures: Stability a Key Issue. Clinical Orthopaedics and Related Research, 468, 243-251. http://dx.doi.org/10.1007/s11999-009-0988-2

[13] Taweel, N.R., Raikin, S.M., Karanjia, H.N. and Ahmad, J. (2013) The Proximal Fibula Should Be Examined in All Patients with Ankle Injury: A Case Series of Missed Maisonneuve Fractures. The Journal of Emergency Medicine, 44, e251-e255. http://dx.doi.org/10.1016/j.jemermed.2012.09.016

[14] Van Rensberg, C.J. (2004) Approach to and Management of Acute Ankle Ligamentous Injuries. CME, 22, $112-115$.

[15] Polzer, H., Kanz, K. and Pranz, W. (2012) Diagnosis and Treatment of Acute Ankle Injuries: Development of an Evidence-Based Algorithm. Orthopaedic Review, 4, e5. http://dx.doi.org/10.4081/or.2012.e5

[16] van Dijk, C.N., Lim, L.S., Bossuyt, P.M. and Marti, R.K. (1996) Physical Examination Is Sufficient for the Diagnosis of Sprained Ankles. Journal of Bone and Joint Surgery, 78, 958-962. http://dx.doi.org/10.1302/0301-620X78B6.1283

[17] Stiell, I. (1996) Ottawa Ankle Rules. Canadian Family Physician, 42, 478-480.

[18] Bachmann, L.M., Kolb, E., Koller, M.T., Steurer, J. and ter Riet, G. (2003) Accuracy of Ottawa Ankle Rules to Exclude Fractures of the Ankle and Mid-Foot: Systematic Review. BMJ, 326, 417.

[19] Dowling, S., Spooner, C.H., Liang, Y., et al. (2009) Accuracy of Ottawa Ankle Rules to Exclude Fractures of the Ankle and Midfoot in Children: A Meta-Analysis. Academic Emergency Medicine, 16, 277-287. http://dx.doi.org/10.1111/j.1553-2712.2008.00333.x

[20] Stiell, I.G., Greenberg, G.H., McKnight, R.D., Nair, R.C., McDowell, I. and Worthington, J.R. (1992) A Study to Develop Clinical Decision Rules for the Use of Radiography in Acute Ankle Injuries. Academic Emergency Medicine, 21, 384-390. http://dx.doi.org/10.1016/S0196-0644(05)82656-3 
[21] Brandser, E.A., Berbaum, K.S., Dorfman, D.D., et al. (2000) Contribution of Individual Projections Alone and in Combination for Radiographic Detection of Ankle Fractures. American Journal of Roentgenology, 174, 1691-1697. http://dx.doi.org/10.2214/ajr.174.6.1741691

[22] Egol, K.A., Amirtharajah, M., Tejwani, N.C., Capla, E.L. and Koval, K.J. (2004) Ankle Stress Test for Predicting the Need for Surgical Fixation of Isolated Fibular Fractures. Journal of Bone and Joint Surgery, 86-A, 2393-2398.

[23] Coughlin, M.J. and Saltzman, C.L. (2006) Chapter 3-Imaging of the Foot and Ankle. In: Coughlin, M.J., Mann, R.A. and Saltzman, C.L., Eds., Surgery of the Foot and Ankle, 8th Edition, Elsevier Mosby, 68-96.

[24] Hoshino, C.M., Nomoto, E.K., Norheim, E.P. and Harris, T.G. (2012) Correlation of Weightbearing Radiographs and Stability of Stress Positive Ankle Fractures. Foot \& Ankle International, 33, 92-98. http://dx.doi.org/10.3113/FAI.2012.0092

[25] Gardner, M.J., Demetrakopoulos, D., Briggs, S.M., Helfet, D.L. and Lorich, D.G. (2006) The Ability of the LaugeHansen Classification to Predict Ligament Injury and Mechanism in Ankle Fractures: An MRI Study. Journal of Orthopaedic Trauma, 20, 267-272. http://dx.doi.org/10.1097/00005131-200604000-00006

[26] Nielson, J.H., Gardner, M.J., Peterson, M.G., Sallis, J.G., Potter, H.G. and Helfet, D.L. (2005) Radiographic Measurements Do Not Predict Syndesmotic Injury in Ankle Fractures: An MRI Study. Clinical Orthopaedics and Related Research, \&NA, 216-221. http://dx.doi.org/10.1097/01.blo.0000161090.86162.19

[27] Hsu, C.C., Tsai, W.C., Chen, C.P., Chen, M.J., Tang, S.F. and Shih, L. (2006) Ultrasonographic Examination for Inversion Ankle Sprains Associated with Osseous Injuries. American Journal of Physical Medicine \& Rehabilitation, 85, 785-792. http://dx.doi.org/10.1097/01.phm.0000237875.90254.4e

[28] Musgrave, D.J. and Fankhauser, R.A. (1998) Intraoperative Radiographic Assessment of Ankle Fractures. Clinical Orthopaedics and Related Research, 351, 186-190.

[29] Stoffel, K., Wysocki, D., Baddour, E., Nicholls, R. and Yates, P. (2009) Comparison of Two Intraoperative Assessment Methods for Injuries to the Ankle Syndesmosis: A Cadaveric Study. The Journal of Bone and Joint Surgery (American), 91, 2646-2652. http://dx.doi.org/10.2106/JBJS.G.01537

[30] Franke, J., von Recum, J., Suda, A.J., Grutzner, P.A. and Wendl, K. (2012) Intraoperative Three Dimensional Imaging in the Treatment of Acute Unstable Ankle Syndesmotic Injuries. The Journal of Bone and Joint Surgery (American), 94, 1386-1390. http://dx.doi.org/10.2106/JBJS.K.01122

[31] Takao, M., Ochi, M., Oae, K., Naito, K. and Uchio, Y. (2003) Diagnosis of a Tear of the Tibiofibular Syndesmosis-The Role of Arthroscopy of the Ankle. The Journal of Bone and Joint Surgery (British), 85, 324-329. http://dx.doi.org/10.1302/0301-620X.85B3.13174

[32] Lui, T.H., Ip, K. and Chow, H.T. (2005) Comparison of Radiologic and Arthroscopic Diagnoses of Distal Tibiofibular Syndesmosis Disruption in Acute Ankle Fracture. Arthroscopy: The Journal of Arthroscopic \& Related Surgery, 21, 1370.e1-1370.e7. http://dx.doi.org/10.1016/j.arthro.2005.08.016

[33] Salai, M., Dudkiewicz, I., Novikov, I., et al. (2000) The Epidemic of Ankle Fractures in the Elderly-Is Surgical Treatment Warranted? Archives of Orthopaedic and Trauma Surgery, 120, 511-513.

[34] Lin, C.W., Donkers, N.A., Refshauge, K.M., Beckenkamp, P.R., Khera, K. and Moseley, A.M. (2012) Rehabilitation for Ankle Fractures in Adults. Cochrane Database of Systematic Reviews, 11, Article ID: CD005595.

[35] Wei, S.Y., Okereke, E., Winiarsky, R. and Lotke, P.A. (1999) Nonoperatively Treated Displaced Bimalleolar and Trimalleolar Fractures: A 20-Year Follow-Up. Foot \& Ankle International, 20, 404-407. http://dx.doi.org/10.1177/107110079902000702

[36] Ramsey, P.L. and Hamilton, W. (1976) Changes in Tibiotalar Area of Contact Caused by Lateral Talar Shift. The Journal of Bone and Joint Surgery (American), 58, 356-357.

[37] Hartford, J.M., Gorczyca, J.T., McNamara, J.L. and Mayor, M.B. (1995) Tibiotalar Contact Area. Contribution of Posterior Malleolus and Deltoid Ligament. Clinical Orthopaedics and Related Research, 320, 182-187.

[38] De Souza, L.J., Gustilo, R.B. and Meyer, T.J. (1985) Results of Operative Treatment of Displaced External Rotation-Abduction Fractures of the Ankle. The Journal of Bone and Joint Surgery (American), 67, 1066-1074.

[39] Phillips, W.A., Schwartz, H.S., Keller, C.S., et al. (1985) A Prospective, Randomized Study of the Management of Severe Ankle Fractures. The Journal of Bone and Joint Surgery, 67A, 67-78.

[40] Klossner, O. (1962) Late Results of Operative and Non-Operative Treatment of Severe Ankle Fractures. A Clinical Study. Acta Chirurgica Scandinavica Supplementum, 293, 1-93.

[41] Herscovici Jr., D., Scaduto, J.M. and Infante, A. (2007) Conservative Treatment of Isolated Fractures of the Medial Malleolus. The Journal of Bone and Joint Surgery (British), 89-B, 89-93. http://dx.doi.org/10.1302/0301-620X.89B1.18349

[42] Naqvi, G.A., Cunningham, P., Lynch, B., Galvin, R. and Awan, N. (2012) Fixation of Ankle Syndesmotic Injuries: 
Comparison of Tightrope Fixation and Syndesmotic Screw Fixation for Accuracy of Syndesmotic Reduction. American Journal of Sports Medicine, 40, 2828-2835.

[43] Schepers, T. (2011) To Retain or Remove the Syndesmotic Screw: A Review of Literature. Archives of Orthopaedic and Trauma Surgery, 131, 879-883. http://dx.doi.org/10.1007/s00402-010-1225-x

[44] Makwana, N.K., Bhowal, B., Harper, W.M., et al. (2001) Conservative versus Operative Treatment for Displaced Ankle Fractures in Patients over 55 Years of Age. Journal of Bone and Joint Surgery, 83, 525-529. http://dx.doi.org/10.1302/0301-620X.83B4.11522

[45] Thieme Publications (2005) Ankle Fractures: Nonoperative versus Operative Treatment in the Elderly. Orthopedic Trauma Directions, 3, 9-17. http://dx.doi.org/10.1055/s-2005-919133

[46] Van Schie-Van der Weert, E.M., Van Lieshout, E.M.M., De Vries, M.R., Van der Elst, M. and Schepers, T. (2012) Determinants of Outcome in Operatively and Non-Operatively Treated Weber-B Ankle Fractures. Archives of Orthopaedic and Trauma Surgery, 132, 257-263. http://dx.doi.org/10.1007/s00402-011-1397-z

[47] Bauer, M., Bergström, B., Hemborg, A. and Sandegård, J. (1985) Malleolar Fractures: Nonoperative versus Operative Treatment-A Controlled Study. Clinical Orthopaedics and Related Research, 199, 17-27.

[48] Rowley, D.I., Norris, S.H. and Duckworth, T. (1986) A Prospective Trial Comparing Operative and Manipulative Treatment of Ankle Fractures. The Journal of Bone and Joint Surgery (British), 68, 610-613.

[49] Egol, K.A., Tejwani, N.C., Walsh, M.G., Capla, E.L. and Koval, K.J. (2006) Predictors of Short-Term Functional Outcome Following Ankle Fracture Surgery. The Journal of Bone and Joint Surgery (American), 88, 974-979. http://dx.doi.org/10.2106/JBJS.E.00343

[50] Hintermann, B., Regazzoni, P., Lampert, C., Stutz, G. and Gächter, A. (2000) Arthroscopic Findings in Acute Fractures of the Ankle. The Journal of Bone and Joint Surgery (British), 82-B, 345-351. http://dx.doi.org/10.1302/0301-620X.82B3.10064

[51] Thordarson, D.B., Motamed, S., Hedman, T., Ebramzadeh, E. and Bakshian, S. (1997) The Effect of Fibular Malreduction on Contact Pressures in an Ankle Fracture Malunion Model. The Journal of Bone and Joint Surgery (American), 79, 1809-1815.

[52] Lo, E.Y. and Lee, M.A. (2008) New Concepts in the Surgical Management of Ankle Fractures. Orthopedics, 31, 868872.

[53] Johner, R., Joerger, K., Cordey, J. and Perren, S.M. (1983) Rigidity of Pure Lag-Screw Fixation as a Function of Screw Inclination in an in Vitro Spiral Osteotomy. Clinical Orthopaedics and Related Research, 178, 74-79.

[54] Schaffer, J.J. and Manoli II, A. (1987) The Antiglide Plate for Distal Fibular Fixation. A Biomechanical Comparison with Fixation with a Lateral Plate. The Journal of Bone and Joint Surgery (American), 69, 596-604.

[55] Minihane, K.P., Lee, C., Ahn, C., Zhang, L.Q. and Merk, B.R. (2006) Comparison of Lateral Locking Plate and Antiglide Plate for Fixation of Distal Fibular Fractures in Osteoporotic Bone: A Biomechanical Study. Journal of Orthopaedic Trauma, 20, 562-566. http://dx.doi.org/10.1097/01.bot.0000245684.96775.82

[56] Weber, M. and Krause, F. (2005) Peroneal Tendon Lesions Caused by Antiglide Plates Used for Fixation of Lateral Malleolar Fractures: The Effect of Plate and Screw Position. Foot \& Ankle International, 26, 281-285.

[57] Lamontagne, J., Blachut, P.A., Broekhuyse, H.M., O’Brien, P.J. and Meek, R.N. (2002) Surgical Treatment of a Displaced Lateral Malleolus Fracture: The Antiglide Technique versus Lateral Plate Fixation. Journal of Orthopaedic Trauma, 16, 498-502. http://dx.doi.org/10.1097/00005131-200208000-00008

[58] Koval, K.J., Petraco, D.M., Kummer, F.J. and Bharam, S. (1997) A New Technique for Complex Fibula Fracture Fixation in the Elderly: A Clinical and Biomechanical Evaluation. Journal of Orthopaedic Trauma, 11, 28-33. http://dx.doi.org/10.1097/00005131-199701000-00007

[59] Panchbhavi, V.K., Vallurupalli, S. and Morris, R. (2009) Comparison of Augmentation Methods for Internal Fixation of Osteoporotic Ankle Fractures. Foot \& Ankle International, 30, 696-703. http://dx.doi.org/10.3113/FAI.2009.0696

[60] Ostgaard, H.C., Ebel, P. and Irstam, L. (1990) Fixation of Ankle Fractures: Power-Driven Staples Compared with a Routine Method, a 3-Year Follow-Up Study. Journal of Orthopaedic Trauma, 4, 415-419. http://dx.doi.org/10.1097/00005131-199012000-00009

[61] Pritchett, J.W. (1993) Rush Rods versus Plate Osteosyntheses for Unstable Ankle Fractures in the Elderly. Orthopaedic Review, 22, 691-696.

[62] Ngcelwane, M.V. (1990) Management of Open Fractures of the Ankle Joint. Injury, 21, 93-96. http://dx.doi.org/10.1016/0020-1383(90)90062-Y 
Scientific Research Publishing (SCIRP) is one of the largest Open Access journal publishers. It is currently publishing more than 200 open access, online, peer-reviewed journals covering a wide range of academic disciplines. SCIRP serves the worldwide academic communities and contributes to the progress and application of science with its publication.

Other selected journals from SCIRP are listed as below. Submit your manuscript to us via either submit@scirp.org or Online Submission Portal.
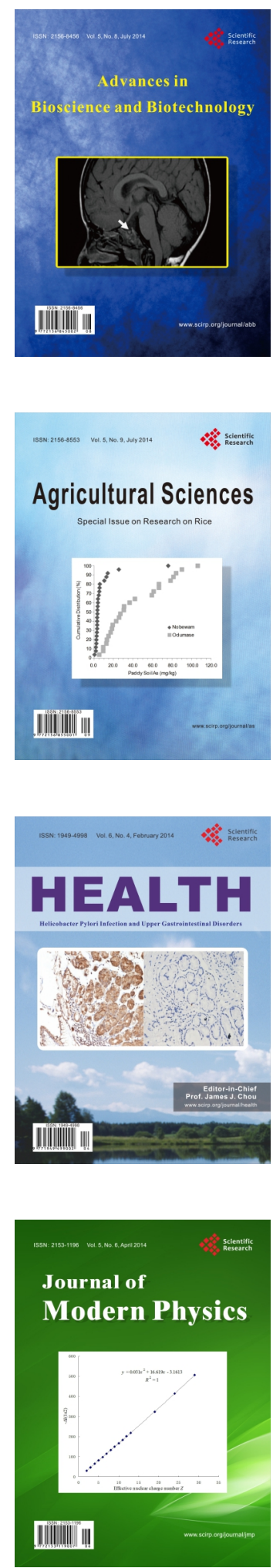
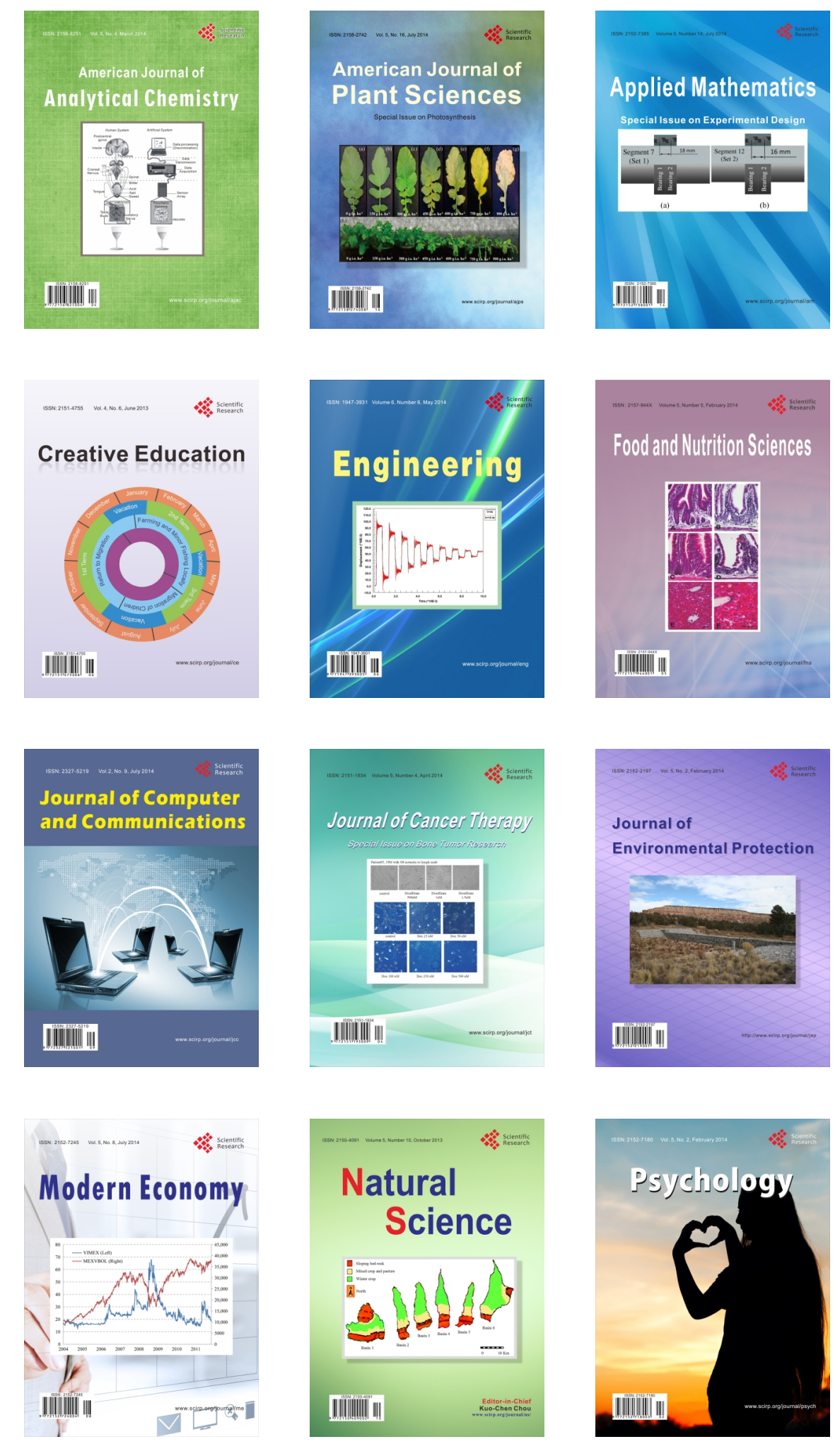